\title{
ORIGINAL
}

\section{CONCENTRACIONES PLASMÁTICAS DE 25-OH VITAMINA D Y PARATHORMONA EN SANGRE DE CORDÓN UMBILICAL}

\author{
Sandra Ortigosa Gómez (1), Oscar García-Algar (1,2), Antonio Mur Sierra (1,2), Roser \\ Ferrer Costa (3), Antonio Carrascosa Lezcano (3,2) y Diego Yeste Fernández $(4,2)$.
}

(1) Servicio de Pediatría, Hospital del Mar. Barcelona. España.

(2) Universitat Autònoma de Barcelona. Barcelona. España.

(3) Laboratorio de hormonas. Hospital Vall d'Hebron. Barcelona. España.

(4) Servicio de Pediatría. Hospital Vall d’Hebron. Barcelona. España.

\section{RESUMEN}

Fundamentos: Los niveles de vitamina D $(25(\mathrm{OH}) \mathrm{D})$ del recién nacido dependen de los depósitos maternos, presentando mayor riesgo de hipocalcemia, raquitismo e infecciones durante el primer año de vida si existe déficit. Recientemente se han publicado estudios que muestran una alta prevalencia de deficiencia de vitamina $\mathrm{D}$ en mujeres gestantes. El objetivo de estudio fue analizar los niveles de 25(OH)D en sangre de cordón umbilical y determinar si existe una relación con factores nutricionales, socioeconómicos y clínicos de las mujeres gestantes.

Métodos: Entre marzo y mayo de 2013 se seleccionó a 99 gestante del Hospital del Mar (Barcelona), en las que se determinaron las concentraciones de $25(\mathrm{OH}) \mathrm{D}$ y parathormona en sangre de cordón umbilical. Se recogieron datos de la historia clínica y se realizó una encuesta sobre ingesta de vitamina D y calcio así como de exposición solar. Se realizó el análisis estadístico mediante el programa SPSS. Las comparaciones se realizaron mediante test de Kruskal-Wallis y U de Mann-Whitney, aplicando corrección por comparaciones múltiples de Bonferroni. Se consideró estadísticamente significativa una $\mathrm{p}<0,05$ y de 0,0083 para comparaciones múltiples.

Resultados. El valor medio de $25(\mathrm{OH}) \mathrm{D}$ en sangre de cordón fue $10,4 \pm 6,1 \mathrm{ng} / \mathrm{ml}$. El $94 \%$ de las mujeres presentaron niveles de $25(\mathrm{OH}) \mathrm{D}$ en sangre de cordón $<20 \mathrm{ng} / \mathrm{ml}$. La ingesta de vitamina D y calcio fueron adecuadas en $92 \%$, aunque la exposición solar resultó deficitaria en $47 \%$. Se encontró una correlación entre niveles de $25(\mathrm{OH}) \mathrm{D}$ e ingesta de vitamina D $(p<0,033)$ y calcio $(p<0,005)$, exposición solar $(p<0,001)$, etnia $(p<0,001)$, fototipo cutáneo $(p<0,001)$ y uso de indumentaria tradicional $(p<0,001)$.

Conclusiones. Existe una elevada prevalencia de déficit de vitamina D en sangre de cordón umbilical tras los meses de invierno. Los niveles de 25(OH)D más bajos se observaron en etnia indopakistaní, fototipo oscuro y baja exposición solar.

Palabras clave: Déficit de Vitamina D. Embarazo. Grupos étnicos. Hormona paratiroidea. Recién nacido. Sangre fetal. Vitamina D.

Correspondencia

Sandra Ortigosa Gómez

Servicio de Pediatría, Hospital del Mar

$\mathrm{P}^{\circ}$ Marítimo 25-29

08003 Barcelona

96722@parcdesalutmar.cat

\section{ABSTRACT \\ Sociodemographic Factors Related to Plasma Concentrations of 25-OH Vitamin D and PTH in Cord Blood}

Background. Plasma 25(OH)D levels in the newborn are dependent on maternal stores, thus, neonates of vitamin D-deficient mothers present a greater risk of hypocalcaemia, rickets and infections the first year of life. Several studies showing a high prevalence of vitamin $\mathrm{D}$ deficiency in pregnan women have been published recently. The aim of the study is to analyze the levels of $25(\mathrm{OH}) \mathrm{D}$ in cord blood and determine whether there is a relation with nutritional, socioeconomic and clinical factors of pregnant women and their newborns.

Metthods. Between March and May 2013, 99 pregnant women were recruited in Hospital del Mar (Barcelona), in whom plasma 25(OH)D and PTH levels were measured in cord blood at birth. Clinical history data were collected and a nutritional survey was made on maternal vitamin D and calcium intake and sun exposure. Statistical analysis was performed using SPSS. Comparisons were performed using Kruskal-Wallis and MannWhitney U tests, and correction for multiple comparisons using Bonferroni. $P$ value $<0.05$ and $<0.0083$ for multiple comparisons were considered statistically significant.

Results. Mean 25(OH)D value in cord blood was $10.4 \pm 6.1 \mathrm{ng} / \mathrm{ml} .94 \%$ of pregnant women had 25(OH)D levels in cord blood $<20 \mathrm{ng} / \mathrm{ml}$. Vitamin $\mathrm{D}$ and calcium intake was considered adequate in $92 \%$ although sun exposure was deficient in $47 \%$. A correlation between serum $25(\mathrm{OH}) \mathrm{D}$ and vitamin $\mathrm{D}(\mathrm{p} 0.033)$ and calcium intake ( $\mathrm{p} 0.005)$, sun exposure $(\mathrm{p}<0.001)$, ethnicity $(\mathrm{p}<0.001)$, skin phototype $(\mathrm{p}<0.001)$ and use of traditional clothing $(\mathrm{p}<0.001)$ was found.

Conclusions. There is a high prevalence of low levels of vitamin D after winter months in cord blood. The lowest $25(\mathrm{OH}$ D levels were observed in Indo-Pakistani ethnicity, dark phototype and deficient sun exposure.

Keywords: Vitamin D deficiency. Pregnancy. Ethnic Groups. Parathyroide Hormone. Newborn. Fetal Blood. Vitamin D. 


\section{INTRODUCCIÓN}

El impacto del déficit de vitamina D durante la gestación, tanto en la salud materna como en la neonatal, ha atraído mucho interés en los últimos años. Se ha sugerido que mantener adecuados niveles de vitamina D en la mujer durante el embarazo es de vital importancia para la salud esquelética y extraesquelética de ambos, madre y recién nacido $^{1-2}$. Estos dependen exclusivamente de los depósitos de vitamina $\mathrm{D}$ de la madre para asegurar un nivel adecuado de vitamina D al nacer. Durante el embarazo el calcio se transporta activamente a través de la placenta de la madre al feto para asegurar el crecimiento esquelético, mientras que la $25(\mathrm{OH}) \mathrm{D}$ se difunde fácilmente a través de la placenta y se almacena en el feto para iniciar el metabolismo de la vitamina D en el neonato, siendo la homeostasis del calcio fetal y el desarrollo esquelético intraútero independiente del estado de vitamina D. Es tras el nacimiento que esta asume su papel regulador del metabolismo cálcico y del desarrollo esquelético ${ }^{3}$.

La correlación entre los niveles de $25(\mathrm{OH}) \mathrm{D}$ en sangre materna y en sangre de cordón está bien demostrada ${ }^{6-14}$, siendo los niveles en sangre de cordón de aproximadamente un $80 \%$ de la sangre materna y en el recién nacido un $60-75 \%$ de los niveles maternos. Esta correlación parece perderse cuando existe una hipovitaminosis materna, siendo los niveles en sangre de cordón incluso más elevados que los maternos ${ }^{5,7,8}$. Estos hallazgos podrían reflejar un esfuerzo por parte de la unidad fetoplacentaria para cubrir las necesidades del recién nacido. Aun así, los últimos estudios sugieren que para un análisis exhaustivo del estado de vitamina $\mathrm{D}$ se deberían determinar las distintas formas de vitamina D incluyendo los epímeros, así como su proteína transportadora, un parámetro significativo de la dinámica de la vitamina D durante el embarazo9.

Dado que la 25(OH)D tiene una vida media corta (2-3 semanas), las concentraciones de vitamina $\mathrm{D}$ en el recién nacido disminuyen rápidamente durante el periodo neonatal a no ser que la provea una fuente exógena. Así, si el estado de vitamina $\mathrm{D}$ materno es pobre, las bajas concentraciones de $25(\mathrm{OH})$ $\mathrm{D}$ en el recién nacido disminuyen rápidamente al rango de deficiencia, pudiendo dar lugar a hipocalcemia o raquitismo durante el período neonatal ${ }^{3,7}$.

Además los depósitos insuficientes de vitamina $\mathrm{D}$ en la madre hacen que los niveles en leche materna sean también bajos, lo que hace que la lactancia materna exclusiva aumente el riesgo de déficit de vitamina $\mathrm{D}$ en el lactante ${ }^{10,11}$.

El mejor indicador del estado de vitamina D es la concentración sérica de $25(\mathrm{OH}) \mathrm{D}$, ya que no está regulada y refleja tanto la ingestión como la síntesis cutánea de vitamina D. Sin embargo no hay un consenso absoluto de cuál debe ser el valor normal de vitamina D. Actualmente, como resultado de numerosos estudios, la mayoría de autores están de acuerdo en considerar valores de déficit los inferiores a $20 \mathrm{ng} / \mathrm{ml}$, insuficientes entre 20 $30 \mathrm{ng} / \mathrm{ml}$ y suficientes por encima de $30 \mathrm{ng} /$ $\mathrm{ml}^{12}$. Existe más controversia con los niveles de vitamina D en las mujeres gestantes, siendo desconocidos los niveles óptimos durante la gestación ${ }^{1,2}$ y en sangre de cordón umbilical, tomándose como referencia los mismos que en la población adulta.

En los últimos años se han publicado varios estudios que muestran una alta prevalencia de deficiencia de vitamina $\mathrm{D}$ en mujeres gestantes ${ }^{4-5,13-17}$.

El déficit de vitamina $\mathrm{D}$ durante el embarazo se ha relacionado con efectos adversos durante la gestación ${ }^{1}$, incluyendo la diabetes mellitus gestacional, el aumento del número de cesáreas ${ }^{18,19}$ y del riesgo de preeclamp$\mathrm{sia}^{20}$. Sobre el recién nacido, los niveles bajos de vitamina $\mathrm{D}$ durante el período fetal y neonatal se han asociado con raquitismo, retraso del crecimiento fetal $1^{1,21,22}$, osteopenia, alteraciones de la osificación fetal, 
craneotabes, hipocalcemia, debilidad muscular, tetania, enfermedades cardíacas y mayor incidencia de infecciones durante la infancia ${ }^{1,2,23-25}$. Nuevas evidencias apuntan a que su déficit crónico aumenta el riesgo de algunos tipos de cáncer, enfermedades cardiovasculares, alteraciones del desarrollo cognitivo $^{23}$, reducción de la masa mineral ósea $^{24}$ y enfermedades autoinmunes o de disfunción del sistema inmunitario (diabetes mellitus tipo 1, artritis reumatoide, enfermedad inflamatoria intestinal y esclerosis múltiple $)^{2,25,26}$, por lo que la deficiencia de vitamina $\mathrm{D}$ durante la gestación podría incrementar la susceptibilidad a dichas enfermedades, aunque se necesitan más estudios para clarificar las repercursiones de los niveles de vitamina $\mathrm{D}$ prenatales ${ }^{27}$.

En el ser humano, la principal fuente de vitamina $\mathrm{D}$ es la síntesis cutánea por la acción de la radiación ultravioleta $\mathrm{B}$ (UVB), la cual representa aproximadamente el 80$90 \%$ de las necesidades del organismo. El resto proviene de una aportación exógena a través de alimentos como el pescado, la yema de huevo o alimentos enriquecidos con la vitamina. Por lo que una radiación solar inadecuada reduce la síntesis de vitamina D. Esta síntesis depende de factores como la edad, el grado de pigmentación cutánea, el de exposición solar, el uso de protectores solares o la cantidad de luz UVB que llega a la superficie terrestre, la cual se afecta por la estación del año, la hora del día, la localización geográfica y la contaminación ${ }^{3,28}$.

En 2010 El Institute of Medicine (IOM) ${ }^{29}$ estableció que $600 \mathrm{UI} /$ día de ingestión de vitamina $\mathrm{D}$ sería adecuada durante el embarazo y la lactancia, aunque el Comité de $\mathrm{Nu}$ trición de la American Academy of Pediatrics sugiere que se debería recomendar una ingestión superior (1.000 UI) para mantener niveles de suficiencia de vitamina $\mathrm{D}$ tanto en la mujer embarazada como en el recién naci$\mathrm{do}^{11}$. Aun así, según una revisión Cochrane reciente se deben realizar más estudios para evaluar el papel de los suplementos de vita- mina D durante el embarazo y saber la dosis segura y efectiva y el régimen de suplementación ${ }^{1-2}$.

El objetivo de este estudio fue analizar los niveles de 25(OH)D en sangre de cordón umbilical y determinar si existe una relación con los factores nutricionales, socioeconómicos y clínicos de las mujeres gestantes y sus recién nacidos.

\section{SUJETOS Y MÉTODOS}

Entre marzo y mayo del 2013 se seleccionó a las mujeres gestantes que dieron a luz en el Hospital del Mar de Barcelona, situado a una latitud de $41^{\circ} 23^{\prime} \mathrm{N}$. El estudio fue aprobado por el Comité Ético de Investigación Clínica del Parc de Salut Mar y se respetaron los acuerdos de la Declaración de Helsinki.

De 284 mujeres que dieron a luz en el período de estudio, participaron y firmaron el consentimiento informado 99.

Se revisaron las historias clínicas de las madres y sus recién nacidos recogiendo datos sobre tipo de parto, complicaciones durante la gestación, edad gestacional, sexo, datos antropométricos al nacimiento, puntuación de Apgar y morbilidades acompañantes.

Además se identificaron los fototipos cutáneos maternos siguiendo la clasificación propuesta por Fitzpatrick ${ }^{30}$. Se analizó la ingestión de calcio, vitamina D y exposición solar mediante la encuesta de Garabédian ${ }^{31}$. La exposición solar se puntuó de 0-9 considerándose deficitaria niveles inferiores a 4 , media 4-7 y satisfactoria $>7$.

Se obtuvieron muestra de sangre de cordón, que fue recogida inmediatamente tras su clampaje. El suero se almacenó a $-18^{\circ} \mathrm{C}$ protegido de la luz hasta el momento del análisis. Posteriormente se determinaron los niveles de 25(OH)D (LIAISON® $25 \mathrm{OH}$ Vitamin D Total Assay) y PTH (LIAISON ${ }^{\circledR}$ 
N-TACT® PTH II Assay) en el laboratorio del Hospital Vall d'Hebron.

Los niveles de 25(OH)D se definieron como déficit grave los inferiores a $10 \mathrm{ng} / \mathrm{ml}$, déficit moderado entre 10 y $20 \mathrm{ng} / \mathrm{ml}$, insuficiencia niveles de $21-29 \mathrm{ng} / \mathrm{ml}$ y suficiencia niveles $\geq 30 \mathrm{ng} / \mathrm{ml}^{12}$.

El análisis estadístico se realizó mediante el programa SPSS. Los datos descriptivos se presentan como valor absoluto y porcentaje para las variables cualitativas y como media y desviaciones estándar para las cuantitativas. Las comparaciones de las variables cuantitativas entre los diferentes grupos étnicos se realizó mediante la dupla de estadígrafos no paramétricos de Kruskal-Wallis y U de Mann-Whitney, aplicando corrección por comparaciones múltiples de Bonferroni. Para las demás variables dicotómicas se utilizó el test de U de Mann-Whitney. Para correlacionar las diferentes variables cuantitativas se utilizó el coeficiente de correlación de Spearman. Se consideraron estadísticamente significativos los valores de $p<0,05$. Ens las comparaciones múltiples por étnia este valor se fijó en 0,0083 , según formulación de Bonferroni.

\section{RESULTADOS}

Se reclutó un total de 99 mujeres gestantes. Sus características se recogen en la tabla 1.

Las determinaciones de ingestión de calcio y vitamina $\mathrm{D}$, la exposición solar así como los niveles de 25(OH)D y PTH en sangre de cordón en el total de la población y agrupados por etnias se presentan en la tabla 2.

El nivel medio de $25(\mathrm{OH}) \mathrm{D}$ en sangre de cordón fue de $10,4 \pm 6,1 \mathrm{ng} / \mathrm{ml}$, con un rango desde indetectable $(<4 \mathrm{ng} / \mathrm{ml})$ hasta $44,1 \mathrm{ng} /$ $\mathrm{ml}$ (tabla 2). En el 57\% de las muestras existía un nivel inferior a $10 \mathrm{ng} / \mathrm{ml}$, en el $37 \%$ el nivel estuvo entre $10-20 \mathrm{ng} / \mathrm{ml}$, en el $5 \%$ entre 21 y $29 \mathrm{ng} / \mathrm{ml}$ y el $1 \%$ presentó niveles superiores a $30 \mathrm{ng} / \mathrm{ml}$ (tabla 3 ).

\begin{tabular}{|c|c|}
\hline \multicolumn{2}{|c|}{$\begin{array}{c}\text { Tabla } 1 \\
\text { Características de la población }\end{array}$} \\
\hline Edad materna (años) & $28,9 \pm 6,7$ \\
\hline Primíparas (\%) & 53,5 \\
\hline Ingesta polivitamínicos (\%) & 10,1 \\
\hline Ganancia de peso $(\mathrm{kg})$ & $12,2 \pm 4,3$ \\
\hline Fumadoras (\%) & 13 \\
\hline $\begin{array}{l}\text { Etnia }(\%) \\
\text { Caucásica } \\
\text { Indopakistaní } \\
\text { Sudamericana } \\
\text { Subsahariana } \\
\text { Magrebí } \\
\text { Gitana } \\
\end{array}$ & $\begin{array}{r}45 \\
24 \\
20 \\
2 \\
3 \\
6 \\
\end{array}$ \\
\hline $\begin{array}{l}\text { Fototipo cutáneo }(\%) \\
\text { I } \\
\text { II } \\
\text { III } \\
\text { IV } \\
\text { V } \\
\text { VI } \\
\end{array}$ & $\begin{array}{r}0 \\
12,1 \\
35,4 \\
19,2 \\
29,3 \\
4\end{array}$ \\
\hline $\begin{array}{l}\text { Estilo de ropa cubierto (cabeza y brazos) } \\
(\%)\end{array}$ & 12,1 \\
\hline $\begin{array}{l}\text { Complicaciones embarazo (\%) } \\
\text { Diabetes gestacional } \\
\text { Toxemia }\end{array}$ & $\begin{array}{l}5 \\
1\end{array}$ \\
\hline $\begin{array}{l}\text { Tipo de parto }(\%) \\
\text { Eutócico } \\
\text { Distócico } \\
\text { Cesárea }\end{array}$ & $\begin{array}{r}64 \\
29 \\
7 \\
\end{array}$ \\
\hline Líquido meconial (\%) & 15 \\
\hline Riesgo de infección (\%) & 36,4 \\
\hline Edad gestacional (semanas) & $39,4 \pm 1,6$ \\
\hline $\begin{array}{l}\text { Sexo recién nacido }(\%) \\
\text { Femenino } \\
\text { Masculino }\end{array}$ & $\begin{array}{l}35 \\
65\end{array}$ \\
\hline Peso al nacimiento $(\mathrm{g})$ & $3.212 \pm 492,4$ \\
\hline Longitud al nacimiento $(\mathrm{cm})$ & $49,5 \pm 2,3$ \\
\hline Perímetro craneal (cm) & $33,82 \pm 1,6$ \\
\hline Apgar 5 minutos & 10 \\
\hline Pérdida bienestar fetal (\%) & 6,1 \\
\hline
\end{tabular}

Por lo tanto, el $94 \%$ de las muestras presentaron valores inferiores a $20 \mathrm{ng} / \mathrm{ml}$ de $25(\mathrm{OH}) \mathrm{D}$, considerados como deficientes en sangre periférica. Las diferencias fueron estadísticamente significativas entre los diferentes grupos étnicos $(\mathrm{p}<0,001)$, también existieron diferencias significativas $(\mathrm{p}<0,0083)$ comparando cada uno de los 


\section{Tabla 2}

Valores de media y desviaciones estándar del aporte de vitamina D, calcio, exposición solar y niveles de 25(OH)D y PTH en sangre de cordón

\begin{tabular}{|l|c|c|c|c|c|c|c|c|}
\hline & Caucásica & Indopakistaní & Sudamericana & Subsahariana & Magrebí & Gitana & Total & $\mathrm{p}$ \\
\hline $\begin{array}{l}\text { Aporte } \\
\text { Vitamina D } \\
\text { (UI/día) }\end{array}$ & $220,6 \pm 95,7$ & $181,5 \pm 105,4$ & $200,5 \pm 91,5$ & $189,3 \pm 106,1$ & $147,6 \pm 21,8$ & $169 \pm 58,1$ & $201,1 \pm 94,6$ & $\mathrm{p}=0,06$ \\
\hline $\begin{array}{l}\text { Ingestión } \\
\text { calcio } \\
\text { (mg/día)* }\end{array}$ & $908,8 \pm 322,3$ & $568,6 \pm 301,9$ & $765 \pm 260,7$ & $951,2 \pm 424,3$ & $860 \pm 466,5$ & $687,1 \pm 189,2$ & $783,2 \pm 328,6$ & $\mathrm{p}=0,002$ \\
\hline $\begin{array}{l}\text { Índice } \\
\text { exposición } \\
\text { solar* }\end{array}$ & $5,4 \pm 2,6$ & $1,9 \pm 1,5$ & $4,5 \pm 2,5$ & $3,5 \pm 3,5$ & $2 \pm 1$ & $4 \pm 2,8$ & $4,2 \pm 2,7$ & $\mathrm{p}<0,001$ \\
\hline $\begin{array}{l}25(\mathrm{OH}) \mathrm{D} \\
\text { (ng/ml)* }\end{array}$ & $13,2 \pm 6,5$ & $7,2 \pm 5,9$ & $9,1 \pm 3,7$ & $9,8 \pm 7,6$ & $6,9 \pm 1,4$ & $8,7 \pm 2,4$ & $10,4 \pm 6,1$ & $\mathrm{p}<0,001$ \\
\hline $\begin{array}{l}\text { PTH } \\
\text { (pg/ml) }\end{array}$ & $5,5 \pm 1,6$ & $6,4 \pm 1,9$ & $7,2 \pm 4,3$ & $6,9 \pm 1,6$ & $6,8 \pm 3,8$ & $6,1 \pm 1,2$ & $6,1 \pm 2,5$ & $\mathrm{p}=0,06$ \\
\hline
\end{tabular}

Tabla 3
\begin{tabular}{|l|c|c|c|c|c|}
\hline \multicolumn{7}{|c|}{$\begin{array}{l}\text { Comparación del déficit de vitamina D en sangre de cordón entre etnias } \\
(\mathbf{p}<\mathbf{0 0 0 1})\end{array}$} \\
\hline & Caucásica & Indopakistaní & Sudamericana & Otras & Total \\
\hline Déficit grave $(<10 \mathrm{ng} / \mathrm{ml})$ & 13 & 20 & 14 & 9 & 56 \\
\hline Déficit moderado $(10-19 \mathrm{ng} / \mathrm{ml})$ & 27 & 3 & 5 & 2 & 37 \\
\hline Insuficiencia $(20-29 \mathrm{ng} / \mathrm{ml})$ & 3 & 1 & 1 & 0 & 5 \\
\hline Suficiencia $(\geq 30 \mathrm{ng} / \mathrm{ml})$ & 1 & 0 & 0 & 0 & 1 \\
\hline TOTAL & 44 & 24 & 20 & 11 & 99 \\
\hline
\end{tabular}

grupos étnicos, excepto las etnias sudamericana e indopakistaní comparadas con los grupos étnicos subsahariano, magrebí y gitano, que se consideraron para los análisis estadísticos como un mismo grupo. La indopakistaní fue la etnia que presentó valores más bajos, con un nivel medio de $7,2 \pm 5,9 \mathrm{ng} / \mathrm{ml}$.

Los niveles de medios de PTH en sangre de cordón umbilical fueron de $6,1 \pm 2,5 \mathrm{pg} / \mathrm{ml}$ (rango $<4-22,4 \mathrm{pg} / \mathrm{ml}$ ). No existieron diferencias estadísticamente significativas entre etnias ni correlación con los niveles de $25(\mathrm{OH})$ D.

En el cuestionario de Garabédian para ingestión de vitamina $\mathrm{D}, 8$ mujeres gestantes mostraron un aporte de vitamina $\mathrm{D}<80 \mathrm{UI} /$ día),
46 un aporte medio (80-200 UI/día) y 45 elevado (>200 UI/día). Si analizamos estas diferencias por etnias (tablas 2 y 4 ) se ve que no existieron diferencias estadísticamente significativas entre ellas $(\mathrm{p}=0,06)$.

La ingestión de calcio (tablas 2 y 4 ) fue considerada adecuada (400-1200 mg/día) en 91 mujeres, siendo baja ( $<400 \mathrm{mg}$ /día) en 8 , en las cuales sí hubo diferencias estadísticamente significativas por etnias con una $\mathrm{p}$ global de 0,002, siendo la etnia indopakistaní la que presentaba una ingestión de calcio inferior, seguida de la sudamericana. Al realizar las comparaciones múltiples se encontró una significación estadística al comparar el grupo indopakistaní con el caucásico $(\mathrm{p}<0,001)$. 


\begin{tabular}{|c|c|c|c|c|c|}
\hline \multicolumn{6}{|c|}{$\begin{array}{c}\text { Tabla } 4 \\
\text { Comparación de la ingestión de vitamina } \mathrm{D} \text {, calcio y nivel de exposición solar } \\
\text { entre etnias }\end{array}$} \\
\hline & Caucásica & Indopakistaní & Sudamericana & Otras & Total \\
\hline \multicolumn{6}{|c|}{ Ingesta vitamina $D(p=0,06)$} \\
\hline Baja $(<80$ UI/día) & 2 & 5 & 1 & 0 & 8 \\
\hline Media (80-200 UI/día) & 18 & 9 & 10 & 9 & 46 \\
\hline Alta (>200 UI/díal) & 24 & 10 & 9 & 2 & 45 \\
\hline \multicolumn{6}{|l|}{ Ingesta calcio $(p=0,002)$} \\
\hline Baja (< 400 mg/día) & 0 & 7 & 1 & 0 & 8 \\
\hline Media (400-1200 mg/día) & 38 & 17 & 18 & 10 & 83 \\
\hline Alta (>1200 mg/día) & 6 & 0 & 1 & 1 & 8 \\
\hline \multicolumn{6}{|c|}{ Exposición solar $(p<0,001)$} \\
\hline Deficitaria & 9 & 22 & 8 & 8 & 47 \\
\hline Medio & 15 & 1 & 5 & 1 & 22 \\
\hline Satisfactorio & 20 & 1 & 7 & 2 & 30 \\
\hline
\end{tabular}

En cuanto a la exposición solar (tablas 2 y 4) el 47,5\% de la población fue deficiente, $22,2 \%$ tuvo una exposición media y $30,3 \%$ satisfactoria. La exposición solar varió por etnias $(p<0,001)$, siendo las más expuestas la caucásica, con una puntuación de $5,4 \pm 2,6$, seguida de la sudamericana $(4,5 \pm 2,5)$ y otras $(3,4 \pm 2,5)$ y finalmente la indopakistaní $1,9 \pm 1,5$. Al realizar la comparación por grupos existían diferencias significativas al comparar la etnia indopakistaní con la caucásica y la sudamericana $(\mathrm{p}<0,001)$. El $12,1 \%$ de la población vestía indumentaria tradicional, llevando un estilo de ropa cubierto (cabeza y brazos), presentando todas ellas déficit de vitamina $\mathrm{D}$.

Se encontró una correlación entre los niveles de $25(\mathrm{OH}) \mathrm{D}$ y la ingesta de vitamina $\mathrm{D}(\mathrm{p}=0,033)$ y calcio $(\mathrm{p}=0,005)$, la exposición solar $(\mathrm{p}<0,001)$, el fototipo cutáneo $(\mathrm{p}<0,001)$ y el uso de indumentaria tradicional $(\mathrm{p}<0,001)$.

No se encontró correlación entre los niveles de $25(\mathrm{OH}) \mathrm{D}$ y la ingestión de polivitamínicos (contenido de vitamina D
200 UI) $(p=0,09)$ ni tóxicos durante el embarazo $(\mathrm{p}=0,31)$, enfermedad crónica $(p=0,93)$, complicaciones $(p=0,78)$, tipo de parto $(\mathrm{p}=0,63)$, pérdida del bienestar fetal $(\mathrm{p}=0,65)$, antropometría al nacimiento $(\mathrm{p}=0,6)$, edad gestacional $(\mathrm{p}=0,9)$ ni riesgo de infección $(\mathrm{p}=0,86)$. Sí que se encontraron unos niveles más bajos de $25(\mathrm{OH}) \mathrm{D}$ en las mujeres multíparas $(\mathrm{p}<0,001)$ o en las que habían presentado abortos previos $(p=0,006)$. No existieron diferencias entre grupos étnicos en las distintas variables analizadas.

\section{DISCUSIÓN}

Los resultados de este estudio revelan unos bajos niveles de vitamina $\mathrm{D}$ en sangre de cordón umbilical en una región soleada de Europa que presenta una ingestión moderada de alimentos con vitamina D. Estos resultados reflejan datos previos de déficit e insuficiencia de vitamina D en otros países de Europa y América ${ }^{4-5,13-17,32}$, que han mostrado también un gran porcentaje de niveles subóptimos en mujeres embarazadas y sus recién nacidos. Este déficit es muy variable 
de unos estudios a otros, desde el $20 \%$ de déficit de vitamina $\mathrm{D}^{5}$, hasta un $90 \%$ en Turquía ${ }^{4}$. Nuestro estudio muestra que el déficit de vitamina $\mathrm{D}$ es común tras el invierno en nuestro entorno, siendo de los porcentajes más altos encontrados en la literatura.

Los porcentajes de concentraciones de $25(\mathrm{OH}) \mathrm{D}$ en suero fueron inferiores a $20 \mathrm{ng} /$ $\mathrm{ml}$ casi en la totalidad de los casos. Los valores de corte utilizados para definir el estado de vitamina $\mathrm{D}$ en población general adulta podrían no reflejar exactamente el nivel de vitamina D durante el embarazo ${ }^{1,2}$ y en sangre de cordón. Sin embargo, dado que no hay un nivel de corte definido para niveles en sangre de cordón, se utilizó el de la población general como se sugiere en la literatura.

El consumo inadecuado de calcio o vitamina $\mathrm{D}$ durante la gestación, el estilo de vestir cubierto, el color oscuro de piel, el invierno, la contaminación, la evitación de la exposición solar, el uso de cremas de protección solar o la ausencia de alimentos enriquecidos con vitamina D y la falta de suplementos se han mostrado como los principales factores maternos de déficit de vitamina $\mathrm{D}^{3,29}$.

En nuestro estudio, la ingestión de vitamina D y calcio es adecuada en la mayoría de las mujeres pero, aun así, se detecta un déficit muy elevado de $25(\mathrm{OH}) \mathrm{D}$, por lo que es obvio que la síntesis cutánea de vitamina $\mathrm{D}$ es la principal fuente de vitamina $\mathrm{D}$ del organismo y que aparentemente la exposición solar recibida en nuestro entorno durante el invierno, ya sea por el estilo de vida, las pocas horas pasadas en el exterior, el uso de protección solar, la contaminación de áreas industrializadas o el estilo de vestir no es suficiente para cubrir las necesidades de vitamina $\mathrm{D}$ durante el embarazo.

Una de las limitaciones de nuestro estudio es que no refleja las diferencias estacionales, ya que se llevó a cabo en primavera y, como se ha demostrado en otros estudios, los valores de vitamina $\mathrm{D}$ varían con la estación del año, con lo que seguramente encontraríamos niveles más elevados tras los meses de verano ${ }^{3,32,33}$, razón por la cual sería necesario nueva recogida de muestras a lo largo de todo el año para determinar si existen diferencias estacionales.

Además debemos poner especial interés, como hemos observado en nuestro estudio, en el caso de parte de la población inmigrante que además de tener un fototipo cutáneo más oscuro y mayor déficit de ingestión de calcio, tienen un estilo de vestir tradicional que cubre gran parte del cuerpo debido a sus principios étnicos y religiosos, lo que puede resultar en una restricción más acentuada de la síntesis de vitamina $\mathrm{D}^{4-5,17,33,34}$.

En nuestra investigación no se observa relación entre las concentraciones de $25(\mathrm{OH}) \mathrm{D}$ y la edad, tipo de parto, ganancia ponderal y los parámetros antropométricos al nacimiento, de forma similar a otros estudios realiza$\operatorname{dos}^{4-5}$. Aunque sí que en algún estudio se ha comprobado que con un déficit grave de vitamina $\mathrm{D}$ los recién nacidos pueden presentar menor longitud, peso y perímetro craneal ${ }^{1}$. También se ha visto que la obesidad materna se correlaciona con niveles más bajos de vitamina $D$ en recién nacidos ${ }^{35}$. En nuestro caso no la encontramos, aunque podría ser que al presentar la mayoría unos niveles bajos de 25(OH)D no se pudo comparar con recién nacidos con niveles adecuados de 25(OH)D.

Tampoco se observa correlación con los niveles de parathormona (PTH) en sangre de cordón umbilical. En estudios en los que se han determinado también niveles de PTH, tampoco se ve una elevación junto al déficit de vitamina $\mathrm{D}^{5,34}$, lo que podría significar que durante la gestación los niveles de PTH no dependen de los de vitamina $\mathrm{D}$.

Sí se revelan valores más bajos de $25(\mathrm{OH}) \mathrm{D}$ en las mujeres multíparas o con abortos previos, lo que coincide con un estudio realizado en Estados Unidos en el que las concentraciones de $25(\mathrm{OH}) \mathrm{D}$ se correlacionaban inversamente con la paridad ${ }^{33}$. 
La hipótesis es que el aumento de las necesidades de vitamina $\mathrm{D}$ durante el embarazo podría deteriorar el estado subóptimo de las madres y la multiparidad podría llevar a una incapacidad para recuperar los niveles de vitamina D entre embarazos. Sin embargo los hallazgos de otros estudios similares ${ }^{4-6}$ no son consistentes.

Como hemos comentado, el paso transplacentario de $25(\mathrm{OH}) \mathrm{D}$ es la única fuente de vitamina $\mathrm{D}$ para el feto. Un déficit materno durante la gestación conlleva bajas reservas en el recién nacido, por lo tanto las mujeres embarazadas necesitan tener niveles adecuados de vitamina $\mathrm{D}$ en el momento de dar a luz para asegurar unos niveles suficientes de esta vitamina a sus hijos, por lo menos durante las primeras semanas o meses de vida, dado que la lactancia materna y la evitación de la exposición solar en recién nacidos normalmente hace que no obtengan unos niveles adecuados de dicha vitamina. En nuestro estudio no hemos podido documentar las consecuencias clínicas de este déficit en el recién nacido, por lo que se requerirían otros que estudien la asociación de dicho déficit con las consecuencias clínicas, tanto a corto como a largo plazo.

La elevada prevalencia de deficiencia de vitamina $\mathrm{D}$ en sangre de cordón umbilical al final de la gestación, y en consecuencia en los recién nacidos tras los meses de invierno, asociada con factores sociodemográficos de nuestro entorno, hace plantearnos la necesidad de realizar programas efectivos durante el embarazo de profilaxis con vitamina D, aunque serían necesarios más estudios para determinar cuál sería el tipo de actuación más adecuada.

En conclusión, el déficit de vitamina D presenta una fuerte correlación con la escasa exposición solar, el origen étnico indopakistaní, así como con el fototipo cutáneo oscuro, por lo que se debería considerar a estas mujeres como grupo de riesgo y realizar un control más exhaustivo durante sus gestaciones.

\section{AGRADECIMIENTOS}

Los autores agredecen la contribución de los participantes en el estudio, al Servicio de Obstetricia y Ginecología del Hospital del Mar, especialmente a las matronas y auxiliares de enfermería, su contribución al estudio mediante la recogida de muestras y al personal del laboratorio de urgencias del Hospital del Mar por permitir la conservación de las muestras hasta su procesamiento.

\section{BIBLIOGRAFÍA}

1. De-Regil LM, Palacios C, Ansary A, Kulier R, Peña-Rosas JP. Vitamin D supplementation for women during pregnancy. Cochrane Database Syst Rev. 2012;2:CD008873.

2. Lerch C, Meissner T. Interventions for the prevention of nutritional rickets in term born children. Cochrane Database Sust Rev. 2007;4:CD006164.

3. Thandrayen K, Pettifor JM. Maternal vitamin D status: implications for the development of infantile nutritional rickets. Rheum Dis Clin North Am. 2012;38:61-79.

4. Halicioglu O, Aksit S, Koc F, Akman SA, Albudak E, Yaprak I et al. Vitamin D deficiency in pregnant women and their neonates in springtime in western Turkey. Paediatr Perinat Epidemiol. 2012;26:53-60

5. Nicolaidou P, Hatzistamatiou Z, Papadopoulou A, Kaleyias J, Floropoulou E, Lagona E et al. Low vitamin D status in the mother-newborn pairs in Greece. Calcif Tissue Int. 2005; 78:337-42.

6. Vio Streym S, Kristine Moller U, RejnmarkL, Heickendorff L, Mosekilde L, Vestergaard P. Maternal and infant vitamin D status during the first 9 months of infant life-a cohort study. Eur J Clin Nutr. 2013;67:1022-8.

7. Lee JM, Smith JR, Philipp BL, Chen TC, Mathieu J, Holick MF. Vitamin D deficiency in a healthy group of mothers and newborn infants. Clin Pediatr (Phila). 2007;46:42-4.

8. Salle BL, Delvin EE, Lapillonne A, Bishop NJ, Glorieux FH. Perinatal metabolism of vitamin D. am J Clin Nutr. 2000;71:1317S24S.

9. Karras SN, Shah I, Petroczi A, Goulis DG, Bili H, Papadopoulou $\mathrm{F}$ et al. An observational study reveals that neonatal vitamin $\mathrm{D}$ is primarily determined y maternal contributions: implications of a new assay on the roles of vitamin D forms. Nutr J. 2013;12:77-84.

10. Thiele DK, Senti JL, Anderson CM. Maternal vitamin d supplementation to meet the needs of the breastfed infant: a systematic review. J Hum Lact. 2013;29:16370 
11. Wagner CL, Greer FR; American Academy of Pediatrics Section on Breastfeeding; American Academy of Pediatrics Committee on Nutrition. Prevention of rickets and vitamin D deficiency in infants, children, and adolescents. Pediatrics. 2008;122:114252 .

12. Holick MF, Binkley NC, Bischoff-Ferrari HA, Gordon CM, Hanley DA, Heaney RP et al. Evaluation, treatment, and prevention of vitamin $\mathrm{D}$ deficiency: an Endocrine Society clinical practice guideline. J Clin Endocrinol Metab. 2011;96:1911-30.

13. Newhook LA, Sloka S, Grant M, Randell E, Kovacs CS, Twells LK. Vitamin D insufficiency common in newborns, children and pregnant women living in Newfoundland and Labrador, Canada. Matern Child Nutr. 2009;5:186-9.

14. Bodnar LM, Simhan HN, Powers RW, Frank MP, Cooperstein E, Roberts JM. High prevalence of vitamin D insufficiency in black and white pregnant women residing in the nortern United States and their neonates. J Nutr. 2007;137:447-52.

15. Sachan A, Gupta R, Das V, Agarwal A, Awasthi PK, Bhatia V. High prevalence of vitamin D deficiency among pregnant women and their newborns in northern India. Am J clin Nutr. 2005;81:1060-4.

16. Magbooli Z, Hossein-Nezhad A. Shafaei AR, Karimi F, Madani FS, Larijani B. Vitamin D status in mothers and their newborns in Iran. BMC Pregnancy Childbirth. 2007;7:1.

17. Van der Meer IM, Karamali NS, Boeke AJ, Lips P, Middelkoop BJ, Verhoeven I et al. High prevalence of vitamin $\mathrm{D}$ deficiency in pregnant non-Vestern women in The Hague, Netherlands. 2006;84:350-3.

18. Poel YH, Hummel P, Lips P, Stam F, van der Ploeg T, Simsek S. Vitamin D and gestational diabetes: a systematic review and meta-analysis. Eur J Intern Med. 2012;23:465-9.

19. Scholl TO, Chen X, Stein P. Maternal vitamin D statys and delivery by cesarean. Nutrients. 2012;4:219-30.

20. Bordnar LM, Catov JM, Simhan HN, Holick MF, Powers RW, Roberts JM. Maternal vitamin D deficiency increases the risk of preeclampsia. J Clin Endocrinol Metab. 2007;92:3517-22.

21. Burris JJ, Rifas- Shiman SL, Camargo CA Jr, Litonjua AA, Huh SY, Rich-Edwards JW et al. Plasma 25-hydroxyvitamin D during pregnancy and small-forgestational age in black and white infants. Ann Epidemiol. 2012;22:581-6.

22. Brunvand L, Quigstad E, Urdal P, Haug E. Vitamin D deficiency and fetal growth. Early Hum Dev. 1996;45:27-33.
23. Whitehouse AJ, Holt BJ, Serralha M, Holt PG, Kusel MM, Hart PH. Maternal serum vitamin D levels during pregnancy and offspring neurocognitive development. Pediatrics. 2012;129:485-93.

24. Javaid MK, Crozier SR, Harvey NC, Gale CR, Dennison EM, Boucher BJ et al. Maternal vitamin D status during pregnancy and childhood bone mass at age 9 years: a longitudinal study. Lancet. 2006;367:36-43.

25. Hollick MF. Vitamin D: importance in the prevention of cancers, type 1 diabetes, heart disease, and osteoporosis. Am J Clin Nutr. 2004;79:362-71.

26. Misra M, Pacaud D, Petryk A, Collett-Solberg PF, Kappy M, Drug and Therapeutics Committee of the Lawson Wilkins Pediatric Endocrine Society. Vitamin $\mathrm{D}$ deficiency in children and its management: review of current knowledge and recommendations. Pediatrics. 2008;122:398-417.

27. Lucas RM, Ponsonby AL, Pasco JA, Morley R. Future health implications of prenatal and early-life vitamin D status. Nutr Rev. 2008;66:710-20.

28. Masvidal Aliberch RM, Ortigosa Gómez S, Baraza Mendoza MC, Garcia-Algar O. Vitamina D: fisiopatología y aplicabilidad clínica en pediatría. An Pediatr (Barc). 2012;77:279.

29. Institute of Medicine of the National academies. Dietary reference intakes of calcium and vitamin D. [citado el 26 Marzo 2013]. Disponible en: http://www. iom.edu/Reports/2010/Dietary-Reference-Intakes-forCalcium-and-Vitamin-D.aspx 30. Fitzpatrick, T. B. Soleil et peau. J de Medecine Esthetique. 1975;2: 33-34

31. Garabédian M, Menns S, Nguyen TM, Ruiz JC, Callens A, Uhrich J. Prévention de la carence en vitamina d chez lénfant et ládolescent I. Proposition et argumentarie pour lútisitaion d'un abaque décisionnel. Arch Pédiatr 1999;6:990-1000.

32. McAree T, Jacobs B, Manickavasagar T, Sivalokanathan s, Brennan L, Basset P et al. Vitamin D deficiency in pregnancy - still a public health issue. Matern Child Nutr. 2013;9:23-30.

33. Eichholzer M, Platz EA, Bienstock JL, Monsegue D, Akereyeni F, Hollis BW et al. Racial variation in vitamin $\mathrm{D}$ cord blood concentration in white and black male neonates. Cancer Causes Control. 2013;24:91-8.

34. Sulaiman RA, Sharratt CL, Lee PW, Skinner A, Griffiths MJWbster C et al. Ethnic differences in umbilical cord blood vitamin $\mathrm{D}$ and parathyroid hormone - south Asians compared to Whites born in the UK. J Matern Fetal Neonatal Med. 2010;23:1315-7.

35. Josefson JL, Feinglass J, Rademaker AW, Metzger BE, Zeiss DM, Price HE, Langman CB. Maternal obesity and vitamin D sufficiency are associated with cord blood vitamin D insufficiency. J Clin Endocrinol Metab. 2013;98:114-9. 